\title{
Maurice Blanchot: Littérature et ruine de l'écriture
}

Thierry DURAND

"Tout homme n'existe qu'expulsé de soi-même et en peine d'une réalité qu'il ne peut ajouter qu'à autrui."

Joë Bousquet 321

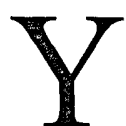

ves Bonnefoy, Henri Meschonnic et Claude Rabant présentent Maurice Blanchot comme le prophète d'une modernité désenchantée, d'une "ère sans naïveté," comme il le dit lui-même dans $L$ 'Amitié (136). Anti-humaniste par excellence, l'écriture est la vanité même et celle de Blanchot s'abîme interminablement dans le désastre qu'elle entretient. C'est que le critique-écrivain ne se veut pas théoricien du langage, il écrit dans le langage et cette lucidité est, selon lui, le malheur même, l'expérience de la pensée prise dans autre chose qu'elle même. La pratique littéraire prend ainsi le pas sur les disciplines. Si "la littérature est cette manière de dire qui dit par la manière" (Le Livre à venir 66), il faut aussitôt ajouter en effet qu'une telle définition ne repose pas sur une distinction préétablie entre le philosophique et le littéraire ou entre une théorie et une pratique. Au cœur de la pensée blanchotienne, on trouve une impossibilité foncière de communiquer sans détour, impossibilité liée à une intériorité clivée, à une dualité originelle. Françoise Collin remarque ainsi qu'il semble impossible de réduire son œuvre à un système sans gravement manquer à son esprit, sans que l'on puisse cependant, il faut l'indiquer aussitôt, attribuer une telle impuissance à une absence de rigueur philosophique, la 
défaillance étant ici, si on peut dire, essentielle. La littérature n'est pas un agrément mais une expérience dont la limite n'est plus déterminée par un savoir, comme si l'écriture remettait en question jusqu'au concept de savoir lui-même. L'écriture blanchotienne est ainsi fascinée par ce qui l'excède et se présente comme un accompagnement de la pensée jusqu'à sa faillite. De fait, rien ne semble pouvoir subsister en définitive et Blanchot attire l'attention de son lecteur sur cette impossibilité liée à la pratique littéraire.

Pourquoi s'obstiner alors, "pourquoi ce mouvement sans espoir, écrit Blanchot, vers ce qui est sans importance" (L'Espace littéraire 228)? Pourquoi écrit-il, pourquoi écrit-on? Si écrire est une souffrance, une passion désastreuse (il n'y a pas d'idylle, de retrouvailles mais un exil perpétuel), pourquoi cette souffrance est-elle digne d'être soufferte? En quoi consiste la dignité de l'écriture? Dans ce travail, nous voudrions montrer que l'exigence liée à l'écriture — ce qui constitue la responsabilité de l'écrivainest une éthique tournée vers l'autre qui consiste (sans pouvoir "consister") en une pratique de l'expiation. Si écrire n'est en fait qu'un infini commentaire de ce qui ne cesse de se dérober et qui accuse en retour ce qui demeure (est écrit)-la "terrible vanité," comme l'appelle Blanchot ( $L$ 'Entretien infini 140)-l'écriture, certes liée chez lui à une certaine imposture, doit cependant "rendre justice" (L'Entretien infini 140) à l'expérience qui est toujours celle de l'autre (Le Pas au-delà 173). Or, ce "rendre justice" est l'expiation même. Devant ce qu'il faut considérer comme une approche nouvelle dans laquelle la littérature est revêtue d'une importance exclusive (qu'elle n'avait pas aux yeux du journaliste des années trente devant l'urgence désignée par Maurras: "la politique d'abord"), nous voudrions situer ce qui fut sans doute une évolution progressive (marquée par ses lectures, ses amitiés et l'histoire) dans une signification fondamentale, une intentionnalité.

\section{Une écriture pénitente}

Dans Le Pas au-delà, Maurice Blanchot se demande pourquoi la "nécessité d'écriture" donne "lieu à rien qui ne paraisse superflu, vain et toujours de trop" (170)? Cette contestation de l'écriture à la fois "nécessaire" et "vaine" ne constitue pas un thème annexe chez le critique mais rend compte d'une approche essentielle de la littérature que partage Georges Bataille pour qui la poésie est une ouverture à "l'excès du désir." Blanchot est ainsi en proie à un "tourment de non-langage" (La Part du feu 255) qui refuse les paradis artificiels, s'oppose à toute vélléité de retour à un âge d'or et s'interdit l'espoir d'un langage adamique, fût-il celui que nourrit la 
hantise de l'enfance. Dans un article qui lui est consacré, Georges Bataille décrit ainsi 1" "effort" de Blanchot:

Bien entendu chacun demeure libre de parler, mais dès lors, il ne peut entrer dans ce royaume où il connaîtrait ce que ne révèle pas le langage, ce dont Maurice Blanchot a parlé dans ses livres, par une sorte d'effort prodigieux et affreux, où il ne lui est donné d'échouer qu'à la limite de ses forces, qui n'est enfin tolérable qu'à une condition: se livrer sans cesse au jugement qui en dénonce l'échec ("Silence et littérature" 175-6).

La littérature se présente en effet chez Maurice Blanchot comme l'expérience de l'écriture comprise elle-même comme celle du langage au sein duquel sourdent deux exigences qui sont à la fois indifférentes et opposées l'une à l'autre. Il y a donc deux paroles chez Blanchot. Ces deux paroles sont d'une part celle de la totalité présente dans le mythe d'une immanence subjective, communautaire ou politique, d'autre part celle de la fascination du neutre; d'un côté une parole de commentaire qui ramène au jour, de l'autre la parole neutre marquée par son infinie négativité. Ce neutre énigmatique n'est pas, il faut le noter, une autre parole. Il n'est pas davantage ce "point de l'horizon" dont parle Breton, mais un excès de parole et il prend chez différents écrivains différentes tournures; chez Louis-René des Forêts, il est cette voix de l'enfant intraitable et chez Roger Laporte la "voix de fin silence." Le neutre est donc tout ce que le poète ne peut dire et qui ruine le déjà-dit, l'impossibilité de vivre dans le repos mythique, une sorte de dire insatiable, un éternel "tiers exclu" selon Lévinas. Il n'est pas ceci ou cela mais un manque à jouir. Il revient donc à l'écrivain, selon Blanchot, de faire l'expérience non empirique du mythe dénoncé, hors les limites de la subjectivité, comme vanité, tromperie, simulation. Blanchot introduit ainsi une dimension tragique dans la littérature qui n'est pas sans rappeler l'opposition décrite par Nietzsche dans $L a$ Naissance de la tragédie entre l'apollinien et le dionysiaque et qui n'ouvre à aucune consolation métaphysique. Cette parenté avec Nietzsche que nous soulignons à plusieurs reprises au cours de ce travail repose avant tout sur une commune approche de l'homme compris comme déchirement ou, ainsi que le dit le philosophe dans La Naissance de la tragédie, comme "dissonance." 
L'expérience de l'écriture revient donc à "expier" (c'est ainsi que Bataille caractérise dans L'Expérience intérieure la spiritualité chez Blanchot (120)) l'autorité d'un sujet du discours (au deux sens du génitif) car la notion ainsi introduite de sujet retombe toujours sous la condamnation du mythe de l'identité, de la présence ou de l'origine. Dans un véritable conflit de la volonté, il faut à la fois se confier au langage et s'en méfier absolument, écrire et ne pas écrire. L'écriture a donc lieu dans une déchirure qui tient à la nature du projet qui l'anime et qui est l'impossibilité même. Le pourquoi écrire se résorbe ainsi tout entier dans un comment écrire puisqu'au désir lié à l'écriture est attachée la faute ou, comme l'écrit Bataille, la trahison (Le Pas au-delà 159). A cet égard, l'inspiration est toujours celle de la ruine du langage, de la sécheresse, de n'avoir rien à dire et d'avoir à le dire et l'impossibilité d'écrire est avant tout l'espace scénique d'un déchirement. Blanchot prend ainsi place dans une lignée d' "expiateurs de l'esprit," pour reprendre l'expression de Nietzsche: c'est, écrit Laporte dans Une Vie, encore une "faute" que "de céder à la nostalgie d'une fin abrupte" (592); Louis-René des Forêts éprouve la même culpabilité devant "l'enfant intraitable." Ecrire engage donc à une certaine fidélité impossible à l'égard d'une vérité persécutée, et le mot "expiation" renvoie à cette expérience, c'est-à-dire à une certaine affectivité de l'écrivain qu'aucune formule ne peut exprimer. Il ne s'agit pas d'abord chez Blanchot d'un concept ou d'une idée de la raison, mais d'une symptomatique de l'écriture qui engage tout l'homme. L'expiation nomme ainsi une certaine phénoménalité de l'écriture qui se distingue par une pathologie: angoisse, fatigue, maladie, faiblesse. Elle marque le corps et l'esprit.

La pratique de l'écriture exige donc une éthique de l'échec qui ne soit pas "relevée" dans une pensée ou une théorie de l'échec. D'où l'importance, notée chez Blanchot mais aussi chez Bataille et Laporte, d'une critique littéraire ou d'une théorie de la littérature qui ne relève pas l'expérience, qui ne la totalise pas mais qui, en s'expiant à son tour, avoue son caractère à la fois fautif et, il faut le noter, inéluctable. On peut donc dire à propos de Blanchot ce que Eugen Fink déclare à l'endroit de Nietzsche: "Ce qu'il est possible de penser est sans aucun doute une fiction" (La Philosophie de Nietsche 211). D'où, ensuite, l'illusion de toute quête autobiographique au sens conventionnel du terme, "car il se pourrait, déclare Blanchot, que tout nom [. . .] fût encore un effet d'impatience" (L'Ecriture du désastre 66-7). Dans Le Pas au-delà, il écrit ainsi en guise d'avertissement: "N'espère pas unifier ton existence [...] par l'écriture qui désunifie" (8). De même, Laporte éprouve l'impossibilité 
de concilier la vie de l'écriture, la véritable "biographie," avec celle du sujet et définit son expérience comme une "thanatographie."

Blanchot approche donc la conscience non pas en terme d'immanence ou de coïncidence, mais par une dualité, un recul dont l'équivalent, au niveau de l'affectivité, est la conscience coupable. A cet égard, l'angoisse dont Blanchot traite dans Faux pas mais aussi dans Le Pas au-delà, est cette tonalité de la conscience en proie à sa condition fautive, toujours en opposition dans la langue, en porte-à-faux, en trop par rapport à ce qu'il y aurait à dire, "out of phase with itself," explique Joseph Libertson (Proximity 327). Blanchot désigne ainsi le rôle imparti à l'écrivain en disant dans La Part du feu que toute son existence "est d'en avoir fini avec l'existence" (270); dans Le Pas au-delà, il confie: "Nous écrivons pour perdre notre nom" (53) et, dans L'Ecriture du désastre: "La seule faute serait de position: c'est d'être 'je"' (89).

La critique blanchotienne s'inscrit par conséquent dans un courant phénoménologique (l'écriture est abordée dans le vécu de la conscience) en dissidence avec tout ego transcendantal et place au centre de sa problématique la question de la littérature entendue comme l'expression la plus authentique de l'homme dont l'existence est alors tournée en question. L'expérience débute ainsi pour l'écrivain dans le cadre somme toute assez traditionnel du voyage spirituel: la vraie vie est ailleurs. Expression qu'il nous faut cependant immédiatement corriger puisqu'en son fond tragique, la question que porte la littérature se présente sous la forme d'un paradoxe: sans doute l'écrivain est voyant, mais ce qui est pressenti au sein de l'expérience est la faute attachée au dévoilement, à la mise en lumière, au "tourment de voir clair" (Lautréamont et Sade 153). Autrement dit, se dresse le procès de la conscience profanatrice comprise comme l'excès du savoir; l'écrivain est de trop. Ce que Blanchot nous invite à croire, c'est qu'au fond de la littérature en tant que pratique d'un langage authentique, est pressentie la fin de la littérature édifiante au sens humaniste du terme. Il faut écrire pour ne pas écrire, penser pour détruire la pensée et c'est ce travail interminable et incontournable (le suicide n'est qu'une tromperie) qui définit l'activité littéraire. Il ne s'agit pas tant de viser un but inatteignable que d'écrire dans cette situation étouffante, invivable: écrire en ne pouvant le faire ni ne pas le faire. La faute est vécue aux confins de l'existence comme une exigence de disparition, comme la conscience de l'excès de la conscience, comme une "différance" qui insiste à la fois sur la dignité de la tâche et sur sa totale vanité.

La pénitence correspond donc chez Blanchot à l'expiation de l'écriture même qui véhicule la contestation. L'écriture partage ainsi le caractère 
démoniaque de la confession dans la mesure où elle est à la fois dénonciation et transgression, à condition cependant d'écarter tout "projet" de transgression: "[ . . .] La transgression [ . . . ] ne continue-t-elle pas de faire allusion à ce qu'il reste de sacré" (Le Pas au-delà 41)? L'écriture devient ainsi sa propre dramatisation, sa propre mimique. A cet égard, ce que Blanchot appelle "récit" peut être compris à la lumière de la lecture derridienne de Mimique de Mallarmé ou encore de ce que le philosophe appelle "l'invagination" (notamment à propos de La Folie dujour dont le premier titre, Un Récit, est révélateur), c'est-à-dire "le jeu multiple d'une scène qui, n'illustrant rien hors d'elle-même, parole ou acte, n'illustre rien" (La Dissémination 236). Laporte s'efforce ainsi d'écrire l'écart (qui constitue son expérience) par rapport à un centre (la "chose," la "crypte," le "tombeau vide") par lequel il pressent être passé et d'où il est appelé (il parle à cet égard de "compassion"). Par rapport à ce centre fictif qui élude toute présence, tout mythe et qui n'est jamais présent lui-même sinon sous la forme de l'imposture, l'écriture et la souffrance qu'elle comporte sont vécues comme de simples simulacres, de simples mimesis de mimesis, de pures fictions ou tricheries, la réalité du sujet étant pressentie comme sa fin impossible, œuvre de sa mauvaise foi. Il n'y a plus ni origine ni destination, "l'accomplissement se résume dans le désir," écrit Derrida, "le désir est (en avance sur) l'accomplissement qui demeure, toujours mimé, un désir, "sans briser la glace"' (La Dissémination 238). On se rappelle à cet égard la définition que René Char donne de la poésie: "l'amour réalisé du désir demeuré désir" (73).

Une telle caractéristique de l'écriture de Blanchot est illustrée non seulement par le contenu et tout un champ sémantique qui tend à expier le "dit," le recours à la dépréciation soutenue de l'acte d'écrire qui ne peut être que "nécessairement grossier" ou fait "grossièrement," comme en tẻmoignent, entre autres, Le Pas au-delà $(136,141)$ et L'Entretien infini (583), mais aussi dans la syntaxe même qui repose essentiellement sur le paradoxe (il faut dire inlassablement et se dédire pour échapper au dit), la tautologie, l'oxymore et une certaine utilisation de la parataxe ou de la juxtaposition de termes séparés par l'énigme d'une relation seulement pressentie (écrire: mourir). Dans Une Voix venue d'ailleurs, la langue semble ainsi se libérer parfois pour aussitôt tourner court, avorter, retomber, le souffle coupé, comme étranglée. On parlera d'une littérature "pénitentielle" ou d'une écriture expiatoire dans la mesure où justement elle est l'exercice, la pratique de la faute en tant que faute, faute qui consiste précisément et inévitablement à la commettre en "voulant" l'expier. Tout Blanchot est là: la vie qui porte la mort et se maintient fautivement en elle. 
Comme le dit Bataille dans un article intitulé "Le rire de Nietzsche," le salut est à la fois incontournable et ce à quoi il ne faut pas recourir.

En considérant l'écriture blanchotienne comme pratique expiatoire, l'on est donc à même de saisir ce que Ricœur appelle le "symbolisme" du "langage de la confession" dont le mode le plus primitif, écrit-il, est la "souillure" et, dans les présentations les plus élaborées, notamment chez les Hébreux et les tragiques grecs, la "relation blessée, entre Dieu et l'homme, entre l'homme et l'homme, entre l'homme et lui-même" (Le Conflit des interprétations 419). Il est donc intéressant de remarquer que Blanchot reprend à plusieurs reprises le symbole de la blessure. Ce symbole est présent notamment dans Thomas l'obscur où il est fait mention de la "blessure de la pensée;" on le trouve également dans son commentaire de Mme Edwarda de Bataille alias Pierre Angélique. Dans L'Ecriture du désastre, Blanchot avoue que "la pression d'autrui" le "blesse" (38) et il définit la subjectivité comme une "opposition blessée" (39).

L'expiation est donc consubstantielle au désir à l'œuvre dans l'écriture. De surcroît, ce dire qu'est l'expiation du dit, parole tourmentée du tourment, est certes symbolique par "nécessité biologique," écrit Fink à propos de Nietzsche (208)--comment pourrait-il en être autrement?-mais repose sur un refus absolu, aussi absolu que la nécessité du symbole, du symbolique pris en charge dans un savoir de lui-même, théorie qui donc serait en l'occurrence celle de la littérature. Si écrire se refuse par conséquent à l'absolution, à ce que le "muthos [soit] sur le chemin du logos" (418), selon la formule de Ricœur, écrire, chez Blanchot, se refuse aussi au "muthos" lui-même. Il s'agit à la fois de démythologiser et de démythiser. Le symbolisme tragique est ici celui du scandale, de l'errance et de l'exil, de l'erreur et du mourir interminable. En ce sens, on peut définir l'écriture blanchotienne comme une écriture scrupuleuse, une écriture rongée par le scrupule. Ecrire consiste à se retrouver de facto en communication avec un au-delà de soi dans un rapport neutre dont la vérité consiste à expier sa propre perversité.

Il apparaît donc que l'écriture revêt le caractère d'une exigence de dépense, d'un désir qu'on ne peut même plus dire "ontologique" (il n'y a plus de savoir, de "logos" pour le dire), d'effacement de l'écriture, de disparition infinie. L'écrivain a attrapé Silène et sait à présent ce qu'il aurait sans doute mieux valu ne pas "savoir." Au sein de ce conflit entre un sujet du discours et l'exigence dont la littérature est porteuse, l'écriture, investie d'une responsabilité, consiste, sans pouvoir "consister," en l'expiation de tout salut, car toujours elle flétrit l'exigence dont son désir est porteur. L'écriture authentique se présente par conséquent comme une 
"morale" (L'Entretien infini 147) qui demeure informulable. Elle instruit l'écrivain sur une sorte de finalité sans fin qui nie son individualité. Ecrire est un devoir tourné vers l'autre innommable, ce que Blanchot nomme "l'indécision de l'être" (L'Entretien infini 147). A partir de l'expérience de l'écriture, de l'être indécis, l'expiation devient donc une clé pour comprendre non seulement la description blanchotienne de l'acte littéraire mais aussi, comme nous allons le voir, l'éthique qui s'en inspire, que ce soit pour des notions telles que la communauté, l'ami ou le communisme. Imaginons ainsi la situation de l'écrivain: "Bouffon sur son tréteau de songes et de mensonges" comme le présente Louis-René des Forêts dans Les Mégères de la mer, il n'est jamais qu'un imposteur. Certes, il pressent le malheur de la condition humaine, mais quand il veut la faire sienne, elle est toujours déjà celle de l'autre. L'écrivain joue ainsi dans l'expérience la scène où c'est l'autre qui souffre, l'autre qui est l'inconsolable. C'est cette impossibilité de l'écriture qui fonde la communauté.

\section{Une éthique introuvable}

L'expérience de l'écriture est donc indissociable d'une éthique qui insiste sur la priorité ontologique de l'autre (ou du moins le pressentiment de cette priorité) et la position excessive du même. Jean Nabert écrit dans Eléments pour une éthique: "L'ordre du devoir contribue à révéler au moi un désir d'être dont l'approfondissement se confond avec l'éthique ellemême." C'est ce qui se produit dans l'écriture blanchotienne: l'approfondissement de l'écriture, ce que Blanchot appelle, dans L'Entretien infini, la "question la plus profonde," porte l'écrivain vers l'autre, exclut le confort d'une prise de position philosophique pour le livrer littéralement à autrui. Chez Blanchot, l'expiation ne consiste en effet en une littérature pénitentielle que pour autant que l'écriture se ronge, qu'elle tourne autour du manque de sincérité de son expiation et dénonce sa mauvaise foi, sa simulation. Pour Blanchot, la lucidité "fait de la vision la plus juste, une vision coupable, coupable de ce qu'elle condamne" (Lautréamont et Sade 116). L'écriture, tout comme l'individu, la subjectivité ou l'intériorité, ne semblent alors exister, s'incarner, que pour être le support de leur propre expiation, de leur impossible responsabilité. Blanchot écrit ainsi que l'on est condamné à soi-même, "afin qu'il y ait encore quelqu'un pour accueillir autrui" (Le Pas au-delà 177). Il parle de cette "présence à laquelle l'on ne peut être présent, mais d'où l'on ne peut s'écarter" (L'Entretien infini 65). Laporte n'écrit, dit-il, que pour ce "je ne sais quoi d'insupportable auquel je demeure lié par serment mais aussi par désir" (Une Vie 587). L'approche 
de l'écriture et de la littérature en général n'est plus alors dans ce qu'elle dit puisqu'elle est "sans principe," mais dans le mouvement éthique, donc expiatoire, de dire; cela est particulièrement remarquable dans Une Vie de Laporte (où le mot d'ordre est "poursuivre") ou dans le commentaire de Blanchot des Chants de Maldoror où ce qui est dit se dit toujours en retard par rapport au dire et où écrire s'avoue comme la lutte du jour à la "frontière d'une nuit." La littérature raconte toujours pour Blanchot l'histoire d'un rendez-vous manqué, d'un retard originel dont la responsabilité incombe à l'écrivain et à laquelle il ne peut répondre. Ainsi, lorsqu'il commente abondamment le caractère élusif de l'éternel retour de Nietzsche (notamment dans L'Entretien infini et Le Pas au-delä), c'est que ce mythe présente par son ambiguïté même l'éthique qui s'attache à l'écriture: "La présence a lieu dans un langage, alors que la cohérence qui est visée a besoin d'un autre langage qui, dans son altérité, révoque la promesse et ruine la parole qui devrait l'accomplir" (Le Pas au-delà 158).

Ainsi, "éprouvant une peine égale à parler et à se taire," (Le Pas audelà 60) Blanchot présente/pressent l'expérience littéraire comme un "rapport" sans termes, comme un mouvement de débordement, de crue qui oppose le récit au discours, la voix narrative à la voix narratrice, l'intransitivité (auto-référentialité) à la transitivité "accusée" de ses essais critiques, le dire au dit. L'écrivain se tient constamment et douloureusement en équilibre entre deux ordres, deux registres de langage. Le commentaire, traduction infinie, se trouve déchiré entre le jour et la nuit; il est perçu comme manquement à un devoir éthique qui engage un pressentiment de la condition humaine qui s'oppose à toute représentation ontologique et qui naît du désir même de l'écrivain qui, tel Orphée, commet la faute inévitable de regarder ce qui ne supporte pas la lumière.

Une telle déchirure au sein de l'écriture toujours fautive affleure dans le choix qui s'impose à Blanchot de présenter sa critique - par conséquent absolument nécessaire en dépit de son caractère absolument excessifen recourant soit à des mythes (Orphée, les sirènes, l'éternel retour du même etc.), soit à des concepts dérégulateurs, tels le détour, le mourir et le neutre, qui demeurent indéfinissables et invitent à une pensée du désastre. Cette tension se manifeste par exemple dans son ouvrage intitulé Une Voix venue d'ailleurs; Blanchot y insiste "sur cette faute qui est de transformer le poème (les poèmes) en une prose approximative" (17) et avoue qu'il voudrait parler dans un langage qui 1'"obsède" et lui fait "défaut" (21). Il se décrit/décrie comme "incapable" (24). En somme, l'écrivain ressemble à l'enfant dont il est dit qu' il "symbolise le commencement dans l'entre-deux" (31) et qu'il doit "expier" sa présence par "la déception, les 
questions vaines, le silence à la fois obtenu et perdu" (39). Surmonter l'énigme de l'être-là revient donc à être voué comme victime expiatoire dans la tension d'un "à la fois". On peut donc parler à propos de Blanchot, comme le fait Eugen Fink à propos de Nietzsche, d'un "pathos tragique" défini "par un dédoublement et une tension antinomique" (La Philosophie de Nietzsche 218). Il importe par ailleurs de remarquer que la dualité vécue dans l'écriture est aussi celle, irrémédiable, qui relie l'écrivain Maurice Blanchot à l'écrivain Louis-René des Forêts. La faute qui hante l'intériorité de l'écrivain est aussi celle qui ruine la parole de commentaire. C'est toujours l'autre-l'ami-qui écrit.

A la parole hégélienne dialectique et totalitaire s'oppose celle du philosophe-poète, parole de fragment qui invoque en vain. La parole hégélienne nomme en effet chez Blanchot le langage compris comme une médiatisation de l'expérience qui, certes, véhicule une relation conflictuelle, mais seulement dans la mesure où le conflit s'inscrit lui-même dans une histoire qui est celle de l'esprit, celle des hommes eux-mêmes vers le savoir absolu, la totalité; Hegel écrit ainsi dans La Phénoménologie de l'esprit que 'l'expérience exprime la nécessité de réaliser ce qui n'est d'abord qu'intérieur et de le révéler, c'est-à-dire de le revendiquer et de le lier à la certitude de soi-même" (305). Cette parole, à la fois incontournable et mythique selon Blanchot, porte donc le positif dans le négatif qui n'est par conséquent jamais sans issue, jamais sans être relevé dans une "certitude de soi-même." Au contraire, quand Blanchot déclare à la suite de Hegel, notamment dans "l'écriture et le droit à la mort," que la parole d'écriture est l'expérience de "la vie qui porte la mort et se maintient en elle," nous sommes invités à pressentir que ce qui fait progresser le dialecticien, la mort conceptuelle (le négatif travaille la pensée), paralyse l'écrivain qui inscrit son écriture, selon le mot de Todorov, dans un devenir psychotique; "seule," écrit Blanchot, "demeure l'affirmation nomade" (Le Pas au-delà 49). La mort de l'écrivain-on se souvient de l'intervention de Paul de Man rapportée dans The Structuralist Controversy à propos de l'opposition entre Hölderlin et Hegel (184-5)—n'est pas la mort conceptuelle du philosophe, mais celle du poète qui fait l'expérience de son impossibilité, celle de commencer ou de finir, celle donc de la naissance. L'histoire hégélienne est celle d'un éclaircissement, d'une maturité, celle, comme le dit Hegel dans la préface de La Phénoménologie de l'esprit de "la pure reconnaissance de soi dans l'absolu être autre." Il s'agit d'une parole circulaire, centripète, historique et déterminée, motivée. A cela s'oppose l'expérience de la littérature de la modernité, de l'écriture neutre. Le neutre en effet, mythe qui n'offre aucun appui, est ce qui se dérobe et 
esquive toute définition; il est l'interminable, le murmure incessant, le ruissellement, le mourir même dans son inachèvement, ce qui "se dérobe en dérobant et dérobant jusqu'à l'acte de dérober" (Le Pas alı-delà 170). Dans le même ouvrage, Le Pas au-delà, Blanchot écrit aussi: "Au neutre le nom sans nom-rien ne répond, sauf la réponse qui défaille, qui a toujours failli répondre et failli à la réponse. ..." (162). Le neutre échappe donc à toute théorisation ou thésaurisation de l'expérience; il appartient à une sorte de théologie mécréante, à ce que Bataille nomme le "non-savoir." L'espace littéraire se situe dans cet égarement. Le tragique doit ainsi être compris comme la double parole qu'incarnent respectivement Nietzsche et Hegel et qu'il ne s'agit en fait ni d'opposer ni de concilier, chacune occupant simultanément toute la place. On retrouve ainsi certains des thèmes qui caractérisent le tragique: le conflit entre deux droits, deux ordres (le "dit" et le "dire"), le "pathéi mathos" (le comprendre comme épreuve et souffrance), la culpabilité liée à l'existence et la notion de proximité dangereuse, d'un langage risqué.

C'est donc dans un contexte semblable, celui d'une logomachie qui ne peut être résolue dialectiquement, que Blanchot pose, à partir de l'écriture, le problème de la justice due à autrui. Ainsi que nous allons le montrer, on peut expliquer de manière satisfaisante la "mécanique" de l'apparition de l'autre dans cette solitude essentielle qu'est l'écriture. Il faut en effet toujours être deux pour parler. Le problème surgit lorsqu'il s'agit de fonder ou de justifier la contrainte du "rendre justice." Inutile de préciser que le service dû à autrui ne passe pas par le sentiment ou l'apitoiement; il n'y a pas d'humanisme du cœur chez Blanchot. Si la littérature en crise, moderne et critique, "libère la pensée de la notion de valeur" ("Qu'en est-il de la critique?" 36), comment faire apparaître autrui comme celui à qui justice doit être rendue? C'est dans cet abîme de la conversion que se perd non seulement la pensée blanchotienne mais aussi toute lecture critique. On peut dire en effet avec Libertson que l'influence de Lévinas est ici prépondérante dans la mesure où, écrit le philosophe, "en tant que moi, je ne suis pas innocente spontanéité, mais usurpateur et meurtrier" (Totalité et infini 56). Blanchot applique ainsi l'expérience de l'écriture, l'écrivain pour qui c'est toujours l'autre qui souffre car lui-même n'est qu'un simulateur, au rapport avec autrui souffrant. Considérons ainsi, au moins en ce qui concerne la mécanique de l'argumentation, que l'écrivain, à l'instar du Lautréamont de Blanchot, "s'ouvre" à autrui au moment même où il saisit son identité comme apparence, simulacre, image, "absence de filiation." Au moment où l'écriture apparaît dans sa vanité même et bée sur le neutre, l'écrivain s'ouvre à autrui comme à l'absolument 
autre. Ne serait-ce pas alors dans une certaine conception de l'illisibilité foncière de l'écriture qu'il faudrait aller chercher autrui? C'est au moment où l'écrivain, expulsé de sa souffrance (de son origine), au moment où il tombe dans le règne de la mauvaise foi, de la tricherie, faisant ainsi retentir un rire tragique qui rappelle les analyses de Bataille, que l'autre (la doublure de l'écrivain dédoublé) apparaît comme l'ami, le souffrant, l'exposé, la fragilité même. Si Blanchot ne réussit pas à poser de manière convaincante la question de l'altérité d'autrui (c'est l'opinion de Libertson), c'est que l'expérience de l'écrivain ne le conduit pas à identifier un "autrui," mais seulement à pressentir le gouffre qui le sépare de l'ami. Dans Une vie, Roger Laporte confie de même: " $M$ 'approcher de l'épreuve m'écarte tellement de moi-même que mon tourment fait place à une souffrance que je supporte, mais dont je ne puis plus affirmer qu'elle est encore la mienne" (586-7). C'est dans ce dédoublement que naît la conscience comme conscience coupable. Ricœur écrit: 'La coulpe, en latin culpa, est l'auto-observation, l'auto-accusation et l'auto-condamnation par une conscience dédoublée" (420). C'est cela l'important: la tragédie de l'écriture rejoue toujours pour la première fois la rencontre manquée avec autrui souffrant. C'est dans cette exigence de la substitution d'une part accusant l'autre, qu'autrui apparaît alors non seulement comme l'irrémédiablement autre, mais aussi comme l'inconsolable, la vérité persécutée; il est celui qu'il faut protéger contre les forces du dehors et celui qu'il est cependant impossible de sauvegarder comme en témoignent George Steiner dans Language and Silence à propos de la relation douloureuse entre le Juif et son fils et, dans un tout autre contexte, le personnage de Steiner dans $L a$ Dolce vita de Fellini. Si l'écriture est ici expiatoire, c'est à nouveau dans le cadre de l'exigence qui s'ensuit d'un sacrifice jamais poussé assez loin, d'une écriture jamais assez passive, qui n'est jamais suffisamment pas assez; Blanchot écrit: "Je le sais, j'existe encore trop, d'un trop peu qui est de trop" (Le Pas au-delà 181). L'écriture conduit l'écrivain à vivre, sur le mode du simulacre, la souffrance tragique comme destin de l'autre et à se trouver ainsi de facto responsable de cette injustice. D'où le devenir "christique," c'est-à-dire de substitution, noté par Daniel Oster. L'écriture est chez Blanchot le pressentiment de cette souffrance sans salut qui toujours est d'abord celle de l'autre car pour répondre, il faut trahir ou plutôt, pour pouvoir être responsable, se sentir concerné par cette responsabilité, il faut avoir ruiné la possibilité d'honorer cette responsabilité qui n'existait pas avant. Il faut passer par une parole dialectique qui s'oppose au neutre sans que le neutre ne s'y oppose: "Il faut avoir passé par la nécessité de l'un pour répondre" (Le Pas all-delà 172-3), comme si 
la connaissance, ajoute Blanchot, ce que Lévinas appelle la "récurrence," "ne nous était laissée que pour connaitre ce que nous ne pouvons supporter de connaitre" (Le Pas au-delà 170). L'éthique ou la communication ou l'écriture (mots qui sont ici synonymes) me fait comparaitre en tant que subjectivité devant l'autre pour que cette subjectivité excessive se voit ensuite investie, accusée, questionnée, "de sorte que toute parole serait morale, selon Blanchot, mais toujours impossible à ressaisir dans la morale" (Le Pas au-delà 69).

\section{L'être juif: la béance de l'écriture}

Le renouveau mythique souhaité par Nietzsche ne trouve donc pas d'écho chez Blanchot. Au contraire, cette possibilité est tout à fait écartée, expiée au profit d'une autre influence, celle du judaïsme compris comme "rejet des mythes." C'est ainsi que se caractérise l'"être juif" selon Blanchot, par "le renoncement aux idoles" ("Les Intellectuels en question" 22 ). Blanchot fait de ce renoncement l'éthique de la relation d'écriture. L'"être juif" prend donc chez Blanchot une double dimension: mode d'être de l'écrivain, il est l'orphelin pour autant que seule l'expérience de l'écriture établit (sans pouvoir l'"établir") une éthique de la communication comprise comme syncope et il est intéressant à ce propos de rapprocher l'analyse de l'“Aleph" dans Le Livre à venir de celle de l'"anacrouse" dans Une Voix venue d'ailleurs. Mais l'écrivain est aussi l'"être juif" en ce que sa parole est l'objet d'une constante persécution de la part de la parole totalitaire.

C'est dans un tel contexte éthique que l'on peut comprendre la communauté telle que Blanchot l'entend et son communisme qui ne doit pas être confondu avec une quelconque stratégie économique ou avec le discours révolutionnaire qui lui-même doit être expié au profit de l'"attente" (Blanchot prend soin dans L'Amitié de distinguer le communisme de tout parti). Le "commun" est au contraire intimement lié à une certaine pratique de l'écriture dont le seul principe, et qui ne peut être ramené à la raison, est à nouveau l'expiation du mythe. Ce point, déclare Nancy dans $L a$ Communauté désauvrée, est celui de "l'interruption du mythe qui nous révèle la nature disjointe ou dérobée de la communauté" (146). Ce que Blanchot appelle "communauté" ou "communisme" dont le fondement est "l'amitié," prend donc son sens dans une certaine compréhension de l'"être-juif" comme "ordre éthique" ("Les Intellectuels en question" 22). La communauté humaine repose sur l'illusion de la substitution, "en accord avec le malheur de tous, ce malheur qui exclut tout malheur" (Le Pas 
au-delà 169) et le communisme repose sur une syncope. Le "gauchisme" de Blanchot doit par conséquent être abordé dans le contexte du questionnement, au sein de la littérature, d'un certain être-ensemble, questionnement qui marque toute son œuvre par l'intermédiaire de la notion de "part." Avoir "partie liée" avec l'exigence communiste se présente ainsi comme une sorte d'impératif porté par le désir à l'œuvre dans l'écriture, hors de portée, une destination pour autant que seule importe l'écriture qui, sans se rapporter, attend. Citons à nouveau Nancy: "Un être singulier [a] très exactement la structure et la nature d'un être d'écriture, d'un être 'littéraire': . . . il s'offre il se tient en suspens" (192). La politique blanchotienne relève donc d'une écriture de la communauté associée à 1'"être juif" que Blanchot définit ainsi: "révélation de la parole comme le lieu où les hommes se tiennent en rapport avec ce qui exclut tout rapport."

Or, à l'image d'autres écrivains et philosophes dont Lévinas, Blanchot voit dans ce silence qui tourmente "car toujours la présence échappe au pouvoir qui fait violence" ( $L$ 'Amitié 247), celui d'Auschwitz, lieu de "disproportion entre la souffrance et toute théodicée," explique Lévinas ("La souffrance inutile" 20) et que Blanchot désigne expressément comme l'impossible origine de toute écriture de la modernité. Ce lieu est donc aussi celui de l'antisémitisme qu'il définit lui-même comme "faute capitale" et manquement à une "exigence métaphysique" (L'Entretien infini 190). L'holocauste, à mettre au passif de la pensée, est en effet cet "écart de temps" dans lequel, désormais dit Blanchot, "nous en viendrions à écrire" (Le Pas au-delà 101). Le "génocide" est ce qui "brûle" la pensée et l'investit. Dans L'Amitié, il désigne ainsi la Shoa comme l" inspiration" de son travail, la "toute-brûlure" (128-9). C'est en dernier lieu vers les camps, une souffrance indicible, une plaie à jamais ouverte, que toute écriture "vouée" trouve son absence d'origine selon lui et la dénonciation du caractère scandaleux de toute fiction:

Ce qui s'est passé là, écrit Blanchot, l'holocauste des Juifs, le génocide contre la Pologne et la formation d'un univers concentrationnaire, est, qu' on en parle ou qu'on n'en parle pas le fond de la mémoire dans l'intimité de laquelle désormais, chacun de nous, le plus jeune comme l'homme mûr, apprend à se souvenir et à oublier. (L'Amitié 128-9)

L'écriture blanchotienne possède ainsi trois caractéristiques: 1) une dénonciation par la littérature de ce qu'il est convenu d'appeler la 
métaphysique occidentale ("Derrière le discours parle le refus de discourir, comme derrière la philosophie parle le refus de philosopher" (Le Pas audelà 143)), c'est-à-dire celle de la valeur totalitaire d'un penser conquérant, dialectique (systématique) dont il faut expier le projet politique (tout projet étant par essence celui d'une épuration), "parler, c'est assujettir" écrit Barthes dans La Leçon-2) une singularisation de ce que Blanchot appelle 1'“être-juif" qui définit l'écriture éthique comme attente et qu'il oppose, avec Lévinas ou Lyotard, à une ontologie de la pureté-3) un certain rapport avec ses activités journalistiques qui ont précédé la deuxième guerre mondiale et dont nous voudrions à présent dire deux mots en guise de conclusion.

\section{Le dernier mot}

A l'image de l'homme, notablement absent de toute mondanité littéraire, le retournement de l'écrivain à la fin des années trente demeure dans une relative obscurité. On pourrait sans doute invoquer l'amitié et l'influence de Lévinas ou encore, au niveau politique et social, le "lâche soulagement" que fut, aux yeux de Léon Blum, le sommet de Münich. D'un côté, on peut donc inscrire l'origine de cette nouvelle écriture dans une biographie. On peut dire avec Bernard-Henri Lévy par exemple, que la conception blanchotienne de la littérature est tournée vers un passé inavouable caractérisé par l'antisémitisme du journaliste des années trente qui écrivit à cette époque de violents articles de politique étrangère à résonance maurrassienne contre, notamment, le gouvernement Blum ou les Républicains espagnols. En réaction contre ce péché de jeunesse, Blanchot serait en proie à "un remords infini" (Les Aventures de la liberté 312). Une telle approche se trouve renforcée par l'importance que l'Holocauste a pris chez le critique et par la relation de la Shoa avec l'éthique liée à la mission de la littérature. Faut-il pour autant, à l'instar de Jeffrey Mehlman et de la polémique déclenchée par La Quinzaine littéraire autour de son article "Littérature et terreur: Blanchot à Combat," finalement publié dans le dernier numéro de $T e l$ Quel, considérer l'œuvre blanchotienne comme une sorte de "cover-up" d'une partie de sa bibliographie? L'article lui-même, s'il réussit à créer un mal à l'aise certain, reprend à son compte un mode de lecture blanchotien qui obombre le point nodal de l'argumentation pour laisser son lecteur en face de rapprochements, de juxtapositions ou de contiguïtés inquiétantes. Il ne s'agit certes pas de remettre en cause l'antisémitisme (modéré par rapport aux délires dont l'époque fut coutumière) du jeune Blanchot mais de s'interroger sur les 
aboutissants de l'article. Si cet article est de fait un tour de force, c'est dans la mesure où il réussit à imposer, de manière presque impressionniste, une certaine continuité entre les deux écritures au prix de la bonne foi du deuxième Blanchot (mais celui-ci ne nous dit-il pas lui-même que la bonne foi doit toujours être questionnée?) car, pour rétablir cette continuité, il faut, écrit Derrida à propos de la polémique déclenchée autour des "early writings" de Paul de Man, "interpréter la discontinuité comme une ruse, consciente ou inconsciente, destinée à dissimuler une persistance ou une subsistance, la répétition têtue d'un projet originaire" (Mémoires pour Paul de Man 212). A la lecture de l'article de Mehlman, on a ainsi l'impression que Blanchot, le précurseur (le père) d'une certaine modernité littéraire, pourrait bien être en fait le point de départ quasi génétique d'une contamination textuelle par un antisémitisme refoulé, une "machine infernale" au cœur de la modernité. Comme Blanchot, Mehlman nous invite à "croire."

Il semble qu'à cet égard la notion de prédestination au malheur d'autrui puisse nous aider à voir plus clair: par là, la première interprétation du retournement, dû lui-même à des événements aussi bien historiques que personnels, événements parmi lesquelles il faut citer, parce que c'est sans doute l'un des plus déterminants, sa rencontre avec Bataille en 1940, se double au sein de l'écriture d'une certaine conception tragique de l'existence qui dépasse l'antisémitisme de jeunesse dévoilé par le biographe. L'écrivain renonce alors à ce qu'il nomme "un absurde désir de salut." L'explication porte désormais et de plus en plus nettement sur l'injustice faite à autrui à partir de la faute toujours déjà commise dans "une corruption du temps" (Spectres de Marx 45), écrit Derrida qui, à propos de l'expiation, ajoute qu'elle est 'innée, donnée 'par' sa naissance autant qu' à' sa naissance" (46). C'est alors dans ce jeu entre le crime de l'autre (dont Auschwitz est le terrible symbole) et celui de l'écrivain qui porte la croix de cette souffrance à laquelle il fut étranger qu'il faut saisir l'écriture comme expérience de l'expiation. C'est la dimension proprement religieuse de l'œuvre blanchotienne. La caractéristique éthique de l'écrivain revient à pressentir au sein de son dédoublement syncopé la fragilité d'autrui en se trouvant investi du devoir de lui porter secours. Dans un article consacré aux intellectuels et publié en 1984 dans Le Débat, il insiste ainsi sur le rôle éthique de l'intellectuel attaché, depuis l'affaire Dreyfus, "essentiellement" à l'"être juif." D'une certaine manière, Blanchot écrivain élève jusqu'au tragique le fait de n'avoir pas su lire l'époque de sa jeunesse; dans sa vie comme dans son œuvre autrui est celui qui est laissé derrière et à qui il faut répondre de cette injustice. Derrida écrit ainsi 
justement qu'“il n'y a tragédie, il n'y a d'essence du tragique qu'à la condition de cette originarité, plus précisément de cette antériorité préoriginaire et proprement spectrale du crime" (Spectres de Marx 46). Pour reprendre cette image hégélienne de la philosophie "fleur suprême" de l'histoire de la dialectique, on peut dire que chez Blanchot la misère de la communication est reprise dans l'écriture pour illustrer dans une différence formelle une même absence de substance. Ce que joue la littérature est la communication comprise comme ce qui fait faute.

Ainsi la "vanité" de l'écriture n'est pas seulement le nihilisme impossible de la fiction. Ou plutôt cette vanité n'est pas sans valeur ou sans signification puisqu'elle est chez Blanchot la pratique de la justice rendue à autrui. Dans l'expérience de l'écriture, l'expiation se présente donc comme un "instant" tragique et s'exerce contre le simple fait d'être-là, libre d'être soi, pour devenir une sorte de structure folle paradoxalement fondatrice de la nature éminemment éthique de l'homme. Blanchot déclare ainsi après Lévinas que "je suis créé responsable, d'une responsabilité antérieure à ma naissance, comme elle est extérieure à mon consentement, à ma liberté, né, par une faveur qui se trouve être une prédestination, au malheur d'autrui, qui est le malheur de tous" (L'Ecriture du désastre 41). Au moment où tout se perd, où il est toujours déjà trop tard, apparaît le visage d'autrui qui appelle au remords-autrui déporté-_comme s'il eût fallu répondre," écrit Blanchot, "à une exigence d'autant plus marquée qu'elle n'exigeait rien d'autre que cette réponse infinie" (Le Pas au-delà 33).

Linfield College 


\section{Notes}

1. Voir Maurice Blanchot et la question de l'écriture (Paris: Gallimard, 1971).

2. La présence de Hegel dans la critique blanchotienne en est un signe. A cet égard, l'écriture se présente comme ce qui, en un autre temps, esquive les exigences d'un penser systématique qui fait retour à soi. Blanchot l'écrit lui-même dans $L$ 'Ecriture du désastre: "quelque chose cloche dans la dialectique" (118). Si, dans les Leçons sur la philosophie de l'histoire, Hegel compare la philosophie à une fleur-"la fleur suprême"-qui fleurit sur les ruines d'une période de l'histoire, image saisissante qui joint, dans une conscience qui sait et domine, la fin d'un monde au savoir d'une renaissance, Blanchot, dans son premier ouvrage de critique littéraire intitulé Comment la littérature est-elle possible? (consacré aux Fleurs de Tarbes de Paulhan), s'intéresse aussi à l'image de la floraison, mais d'une tout autre façon: ce qui fascine ici le critique, "c'est le recommencement sans fin ruinant toute dialectique" ("Qu'en estil de la critique?" (36)), l'interminable négation des fleurs de la rhétorique, la terreur à l'œuvre dans un langage qui ne cesse de s'opposer à lui-même et qui rend impossible toute capitalisation d'un savoir de la littérature devenue alors "contestation de la philosophie," selon l'expression de Michel Foucault, Les Mots et les choses (Paris: Gallimard, 1966), 312. Les fleurs de la rhétorique sont devenues notre horizon indépassable, fleurs "suprêmes" en ce sens.

\section{In L'Impossible in Euvres complètes Vol. III, Préface.}

4. Heidegger écrit ainsi dans sa Lettre sur l'humanisme (Paris: Aubier, 1957): "Le fait de philosopher sur l'échec est séparé par un abîme d'une pensée qui elle-même échoue" (109).

5. Une philosophie par conséquent, mais travaillée par l'autre. La phénoménologie blanchotienne est, en ce sens, bien particulière. Voir à ce propos: Jacques Derrida, Adieu à Emmanuel Lévinas (Paris: Galilée), 95 6. Et Michel Foucault: "L'expérience du phénoménologue est, au fond, une certaine façon de poser un regard réflexif sur un objet quelconque du vécu, sur le quotidien dans sa forme transitoire pour en saisir les significations. Pour Nietzsche, Bataille, Blanchot, au contraire, l'expérience, c'est essayer de parvenir à un certain point de la vie qui soit le plus près possible de l'invivable. Ce qui est requis est le maximum d'intensité et, en même temps, d'impossibilité." "Entretien avec Michel Foucault," Dits et Ecrits (Paris: Gallimard, 1994), 43.

6. La littérature est démoniaque rappelle Bataille: "Non Serviam." 
7. Voir Jacques Derrida, La Dissémination (Paris: Seuil, 1972), 218-9.

8. Jeffrey Mehlman écrit à ce sujet: "Comment un discours critique pourrait-il vraiment 'pénétrer' un texte qui sape à ce point sa propre intériorité, rallier un mode d'écriture si manifestement distante d'ellemême?" "Orphée scripteur:" Poétique 20 (1974), 458.

9. A cet égard, voir l'entretien de Bataille avec Madeleine Chapsal dont une partie est citée par Michel Surya dans Georges Bataille-La Mort à l'cuvve (Paris: Gallimard, 1992), 600.

10. Cité par Ricœur. Le conflit des interprétations, 141.

11. Lévinas, de son côté, déclare: "Comme si en allant vers l'autre je me rejoignais et m'implantais dans une terre, désormais natale, comme si l'éloignement de moi me rapprochait de moi-même déchargé de tout le poids de mon identité. Mouvement dont la poésie serait la possibilité même." "L'être et l'autre. A propos de Paul Celan." Sens et Existence. Hommage à Paul Ricour (Paris: Seuil,1975), 28.

12. Blanchot insiste sur cette absence de principe notamment dans Lautréamont et Sade et L'Ecriture du désastre.

13. L'expression est de Lévinas.

14. "The 'too late' of its spontaneous punctuality implies a prior involvement for which, in some sense, interiority must answer," écrit Joseph Libertson, 323.

15. Cf. la première phrase du Pas au-delà: "Entrons dans ce rapport."

16. On retrouve le même scrupule à propos de René Char dans $L a$ Part du feu, 102.

17. Maurice Blanchot, De Kafka à Kafka (Paris: Gallimard,1981), 1161. Voir aussi $L a$ Part du feu, 293-331.

18. Blanchot écrit ainsi dans Le Pas au-delà: "Mais la folie qui rompt le langage en le laissant apparemment intact ne le laisse intact que pour y accomplir son invisible destruction" (67).

19. "In spite of the prominence of a dissymetrical model of intersubjectivity in his fiction, and in spite of his stable and repeated intuition that the parole is the force or incumbence of differentiation in the general economy, Blanchot has difficulty articulating the concept of nonreciprocity in an intersubjective context" (274). Voir également à ce propos Le Pas au-delà, 173.

20. "N'espère pas te servir des autres pour te libérer de toi," écrit Blanchot dans Le Pas au-delà, 177.

21. Voir Libertson, 324.

22. Cité par Nancy, La Communauté désœuvrée, 187.

23. Voir à cet égard: Celui qui ne peut se servir des mots (Montpellier: Fata Morgana, 1975), 26. 
24. Voir Jeffrey Mehlman, "Blanchot à Combat: Littérature et terreur" in Legs de l'antisémitisme en France (Paris: Denoël, 1984), 21-44.

25. Sur la polémique autour du retournement de Blanchot, voir Maurice Blanchot-Partenaire invisible de Christophe Bident (Editions Champ Vallon, 1998), 540-544.

26. Voir Christophe Bident, 106-109.

27. Dans L'Instant de ma mort, Blanchot rapporte que peu après le débarquement, en 1944, il fut condamné à mort par les occupants pour complicité avec la Résistance alors qu'il se trouvait dans la demeure familiale à Quain, dans la Saône-et-Loire. Sur le point d'être exécuté, il fut sauvé non tant par le maquis venu au secours d'un des leurs, que par le peloton lui-même composé de Russes blancs (l'armée Vlassov) qui virent en Blanchot un jeune "châtelain." Revenu plus tard sur les lieux de son "exécution," le jeune homme se rendit compte que trois paysans avaient été exécutés à sa place. Il est vrai, et c'est cela qui importe, qu'après coup cet instant produit un déplacement non négligeable: plutôt que de trouver l'origine de la critique littéraire blanchotienne dans le trouble de son engagement d'avant-guerre, le critique est ici invité à chercher dans le camp opposé, le bon, une expérience, une raison, aussi insaissable soit-elle, de son esthétique (l'impossible expérience de la mort, l'abîme de la nonorigine, la compassion). Lecture malintentionnée sans doute que je corrigerai ainsi: pour ma part, de ce tout petit livre, je ferai un cri. Blanchot en aurait-il assez de servir d'exemple ou de bouc émissaire dans cette critique continue et ressassante des liens flous, troublants, entre littérature et fascisme? Car enfin il y en a bien d'autres dont le passé n'est guère reluisant et qui ont choisi, eux, de filer dans les couloirs dérobés de l'histoire. Blanchot, quant à lui, n'a rien oublié. Dans ses écrits, on sent partout une conscience morale aiguisée, exacerbée par la sommation de répondre d'un passé qu'elle nous a elle-même appris à lire sans concession, responsabilité dans l'histoire pour ce qui, essentiellement, échappe aux catégories de l'explication historique. Comme Sartre le déclare à propos de Genet, bien que l'instant décisif qui ramasse en lui le choix originel n'ait probablement jamais eu "lieu," la valeur heuristique d'une telle "fiction" (la valeur de ce texte bref et sans indication de lecture-est-ce un récit, un roman, un témoignage?-est en effet de lier dans un seul instant autobiographie et fiction fondatrice) peut cependant nous permettre de retrouver la direction fondamentale de l'œuvre. Si nous lisons aujourd'hui Blanchot avec gravité, sans indulgence, c'est à Blanchot lui-même que nous le devons. Une anecdote que rapporte Le Nouvel Observateur est à cet égard révélatrice: "Dans une lettre publiće le premier novembre dans La Quinzaine littéraire, Blanchot informait Bruno Roy, directeur de la 
maison d'édition Fata Morgana, qu'il refusait désormais de publier dans une maison qui affichait à son catalogue un livre d'Alain de Benoist, fondateur et théoricien de la 'nouvelle droite', directeur de Grèce et cheval de Troie du Front national dans les milieux 'intello'. Bruno Roy a répondu en menaçant Blanchot de révéler son passé d'extrême droite, pourtant déjà connu de tous" ("La vigilance de Blanchot", no.1676, 19-25/12/96, 63). Après s'être dénoncé lui-même, après être devenu l'éternel pénitent, après avoir expié pendant plus de cinquante ans une parole, la sienne, qu'il estime toujours trop prédatrice, après avoir donné cent fois la preuve qu'il n'oubliait rien, Blanchot montre le danger du doigt et on regarde interminablement à qui appartient le doigt. Nous renvoyons à ce propos au livre de Jacques Derrida, Demeure. Maurice Blanchot (Paris: Galilée), 1997.

28. Dans L'Entretien infini, Blanchot écrit à propos d'Auschwitz: "Impossible donc de l'oublier, impossible de s'en souvenir. Impossible aussi, quand on en parle, d'en parler-et finalement comme il n'y a rien à dire que cet événement incompréhensible, c'est la parole seule qui doit le porter sans le dire" $(200$, note 1$)$.

29. Lévinas de son côté écrit: "Ce qui compte, c'est l'idée du débordement de la pensée objectivante par une expérience oubliée dont elle vit." 


\section{Works Cited}

Bataille, Georges. L'Expérience intérieure. Euvres complètes. Vol. V. Paris: Gallimard, 1973.

—. "Silence et littérature." Euvres complètes. Vol. XII. Paris: Gallimard, 1988.

Bident, Christophe. Maurice Blanchot-Partenaire invisible. Seyssel:

Champ Vallon, 1998.

Blanchot, Maurice. La Part du feu. Paris: Gallimard, 1949.

-. Lautréamont et Sade. Paris: Minuit, 1949.

—. L'Espace littéraire. Paris: Gallimard, 1955.

-. Le Livre à venir. Paris: Gallimard, 1959.

-. L'Entretien infini. Paris: Gallimard, 1969.

-.L'Amitié. Paris: Gallimard, 1971.

- Le Pas au-delà. Paris: Gallimard, 1973.

-. L'Ecriture du désastre. Paris: Gallimard, 1980.

—."Qu'en est-il de la critique?" Arguments. Toulouse: Privat, 1983. 1-17.

-. "Les intellectuels en question." Le Débat. Paris: Gallimard, 1984.

-. Une Voix venue d'ailleurs. Plombières-les-Dijon: Cahiers Ulysse, fin de siècle, 1992.

—. L'Instant de ma mort. Montpellier: Fata Morgana, 1994.

Bousquet, Joë. Correspondances. Paris: Gallimard, 1969.

Char, René. "Seuls demeurent." Fureurs et Mystère. Paris: Gallimard, 1967. Derrida, Jacques. La Dissémination. Paris: Seuil, 1972.

- Mémoires pour Paul de Man. Paris: Galilée, 1988.

-. Spectres de Marx. Paris: Galilée, 1993.

-. Adieu à Emmanuel Lévinas. Paris: Galilée, 1997.

-. Demeure. Maurice Blanchot. Paris: Galilée, 1997.

Fink, Eugen. La Philosophie de Nietzsche. Paris: Minuit, 1965.

Foucault, Michel. Dits et Ecrits. Paris: Gallimard, 1994.

Hegel, G.W.F.. La Phénoménologie de l'esprit. Vol. II. Paris: Aubier, 1941. Laporte, Roger. Une Vie. Paris: P.O.L., 1986.

Lévinas, Emmanuel. Totalité et infini. La Haye: Martinus Nijhoff, 1965.

-. "La souffrance inutile." Giornale de metafisica. Vol. IV. Genova: Tilgher, 1982.

Lévy, Bernard-Henri. Les Aventures de la liberté. Paris: Grasset, 1991. Libertson, Joseph. Proximity Lévinas, Blanchot, Bataille and communication. The Hague: Martinus Nijhoff, 1982.

Macksey, Richard, and Eugenio Donato, eds. The Structuralist Controversy. Baltimore: The Johns Hopkins University Press, 1966. 
Nancy, Jean-Luc. La Communauté désœuvrée. Paris: Christian Bourgois, 1986.

Oster, Daniel. “D’un statut d'évangéliste." Littérature 33 (février) 1979.

Ricœur, Paul. Le Conflit des interprétations. Paris: Seuil, 1969. 
